Paediatrica Indonesiana $15: 191 \ldots$ 197. July - August 1975. 191

From the Department of Child Health, Medical School,

University of Indonesia, Jakarta.

\title{
Refeeding with Free Lactose Milk (Al - 110 Nestle) in Children Suffering from Gastroenteritis and Dehydration
}

SUHARJONO, SUNOTO, ASWITHA BOEDIARSO, HENTYANTO HENDARDJI and SOEGIHARTO.

\section{Abstract}

Forty one infants suffering from diarrhoea hospitalized in the Department of Child Health, Medical School, University of Indonesia/Dr. Tjipto Mangunkusumo General Hospital, Jakarta, from August 15, 1973 to January 15, 1974 were refed with "free lactose millk".

The result is as follows :

1. From the point of view of increase of body weight :

$27(65.8 \%)$ out of 41 cases were excellent.

$2(4.9 \%)$ out of 41 cases were good.

$12(29.3 \%)$ out of 41 cases were poor.

2. From the point of view of stopping the diarrhoea :

$25(60.9 \%)$ out of 41 cases were excellent.

$10(24.5 \%)$ out of 41 was good.

$6(14.6 \%)$ out of 41 cases were poor.

Recaived 2nd May 1975. 
Small bowell biopsy from children with gastroenteritis ofiten demonstrates histologically the presence of villious atrophy (Walker Smith, 1969, 1972) which may lead to secondary sugar intolerance (Dahlqvist et al., 1970; Sunoto et al., 1973).

Antibiotic treatment and refeeding with normal milk formula consisting of high content of lactose often gilve unisatisfactory resulits.

In treating Protein Calorie Malnutrittion and/or gastroenteritis in children, a lactose free or low lactose diet might be more reasonable (Suharjono et al., 1971). The purpose of this study is to know the effect of free lactose milk formula on refeeding in children suffering from gastroenteriltis.

\section{Materials and methods}

Forty one children suffering from gastroenterittis and dehyolraition hospitalized in the Department of Child Health Dr. Cipto Mangunkusumo General Hospittal, Jakarta, belonged to this study.

On refeeding after termination of the intravenous fluid therapy, Al-110 (NESTLE) milk formula was orally applied which was free of lactose. The main composition of this formula in 100 grams of powder is as follows:

$\begin{array}{lrl}\text { protein } & 22 \mathrm{gm} \\ \text { fat } & 21 \mathrm{gm} \\ \text { glucose } & 50.8 \mathrm{gm} \\ \text { salt } & 3.3 \mathrm{gm}\end{array}$

water

$2.9 \mathrm{gm}$ calories $\quad 480$ or $670 \mathrm{cal} / \mathrm{L}$.

Starting the realimentation and adaptation we used dilution of $1 / 3$ this formula whilch was increased daily until it reached the proper dilutilon adapted to the requirement of the child.

After 3 consecutive days the proper dilution of Al-110, S.G.M. (a local milk formula product) was substijtuted 2 times daily, Al-110 remained 4 times a day; the following day, further on S.G.M. 4 times and Al-110 2 tumes and usually on the third day mainly S.G.M. formula was given.

The composition of SGM in 100 grams of powder is as follows:

protein $20 \mathrm{gm}$

fat $\quad \mathbf{1 1} \mathrm{gm}$

glucose $28 \mathrm{gm}$

other carbohydrates $22 \mathrm{gm}$

salt $5 \mathrm{gm}$

water $3 \mathrm{gm}$

calories : : 428 or $514 \mathrm{cal} / \mathrm{L}$.

The antibiotic used routinely was Neomycine $50 \mathrm{mg} / \mathrm{kg}$ body weight for 5 consecutive days if leucocytes $2+(10-20$ leucocytes per high power field) were found on microscopic examination of the stools.

Follow up situdy concerning frequency of stools and increase of body weight was carried out for a period of 2 to 3 weeks of hospitalizaltion.

Criteria of the resulits used are as follows:

Increase of body weight is considered excellent if the ratio between mantihly increase of body weight on the Al-110 and the normal standard 
monthly increase according to age is more than 1 . The increase is considered good if the ratio is approximately 1 , and poor if the ratio is less than 1. In evaluating the frequency of the stools the result was regarded as excellent if the stool become already pasty on the second to third day of the formula and diarrhoea did not recur on the introduction of SGM. The result was good if stool become already pasty on the thilrd to fifth day and did not recur or only slightly loose stools appeared on the intro. duction of the SGM. If diarrhoea was still present after fifth day the result was considered poor.

\section{Result}

The result of the trials base on criteria mentioned before can be seen in Table 1. From the results it is revealed that the stopping of diarrhoea was: excellent $25(60.9 \%)$, good 10 $(24.5 \%)$ and poor $6(14.6 \%)$ out of $41(100 \%)$ cases.

The increasing of body weight was: excellent $27(65.8 \%)$, good 2 $(4.9 \%)$ and poor $12(29.3 \%)$ out of $41(100 \%)$ cases.

\section{Discussion}

Disaccharidases are found within the brushborder lining the luminal surface of the intestinal epithelium (Miller and Crane, 1961) and therefare are able to be affected in any disorder in which the intestinal mucose is damaged. Damage of the in- testinal mucosa could result in secondary disaccharide intolerance (Dahlqvist et. al., 1970; Sunoto et al., 1973) ; however this abnormality could be reversible due to the reversibility of the damage of the mucosa in children suffering from gastroenteritis (Walker Smith, 1972, 1973). Normally lactose is present in lower concentration as compared with other brushborder disaccharidases (Dahlqvist et al., 1964) and is also the last to recover completely following damage (Plotkin and Isselbacher, 1964).

Consequently, lactase deficiency is the most important type of secondary dissaccharidase deficiency and therefore free lacitose or low lactose millk formula should be preferably intro duced as primary refeeding in diarrhoeal children. From the present study good to excellent result in refeeding diarrhoeal chilldren with free lactose milk formula are oibviously obtaimed. Only 12 out of $\mathbf{4 1}$ showed poor result in increasing body weight, 3 suffered from PCM, 1 from Down's syndrome and congenital heart disease and 1 from tuberculous meningitis. These accompanying disease might be play a role too in the cause of the poor results.

Twenty seven other patients with excellent results showed increase of body weight of 300 to $750 \mathrm{gm}$ a week.

Companing with the result of previous trial on refeeding with low lactose milk (LLM) produced by a local factory, the difference wras statistically 
TABLE 1: Effeat of free lactose milk (Ai-110 Nestie) on refeeding children suffering from gastro-enteritis and dehydration.

\begin{tabular}{|c|c|c|c|c|c|c|c|c|c|c|}
\hline \multirow{2}{*}{ No. } & \multirow{2}{*}{ Patient } & \multirow{2}{*}{$\begin{array}{l}\text { Age } \\
\text { (mo) }\end{array}$} & \multirow{2}{*}{$\begin{array}{l}\text { Body weight } \\
\text { (B.W.) } \\
\text { on } \\
\text { admission } \\
\text { (gm) }\end{array}$} & \multirow{2}{*}{$\begin{array}{l}\text { Duration } \\
\text { of hospita- } \\
\text { lization } \\
\text { (days) }\end{array}$} & \multirow{2}{*}{$\begin{array}{l}\text { Increase } \\
\text { of B.W. } \\
\text { during } \\
\text { hospitali- } \\
\text { zation } \\
\text { (gm) }\end{array}$} & \multirow{2}{*}{$\begin{array}{l}\text { Period } \\
\text { after } \\
\text { which } \\
\text { diarrhoea } \\
\text { stops } \\
\text { (days) }\end{array}$} & \multirow{2}{*}{$\begin{array}{c}\text { Accompanying } \\
\text { disease }\end{array}$} & \multicolumn{3}{|c|}{$R$ e s u $1 \mathrm{ts}$} \\
\hline & & & & & & & & $\mathbf{E}$ & G & $\mathbf{P}$ \\
\hline 1. & $\mathbf{D}$ & $51 / 2$ & 4300 & 10 & - & 2 & $\begin{array}{l}\text { Tubercolous } \\
\text { meningitis } \\
\text { PCM, Br. Pnia }\end{array}$ & $\mathrm{D}$ & - & $\mathrm{Gr}$ \\
\hline 2. & A. & 11 & 4600 & 7 & 550 & 2 & Br. Pnia & $\mathrm{D}, \mathrm{Gr}$ & - & 一 \\
\hline 3. & $\mathrm{M}$ & 10 & 6300 & 7 & - & 1 & - & $\mathrm{D}, \mathrm{Gr}$ & - & - \\
\hline 4. & $\mathrm{M}$ & $91 / 2$ & 7050 & 8 & 50 & 1 & $\begin{array}{l}\text { Br. Pnia } \\
\text { Convulsions }\end{array}$ & $\vec{D}$ & - & Gr \\
\hline 5. & L & 4 & 4100 & 8 & 350 & 1 & - & $\mathrm{D}, \mathrm{Gr}$ & - & - \\
\hline 6. & $\mathrm{H}$ & 9 & 6900 & 7 & 450 & 1 & - & $\mathrm{D}, \mathrm{Gr}$ & - & - \\
\hline 7. & $\mathrm{~K}$ & 5 & 5300 & 8 & 500 & 1 & 一 & $\bar{D}, \mathrm{Gr}$ & - & - \\
\hline 8. & A & 4 & 3300 & 8 & 380 & 1 & PCM & $\mathrm{D}, \mathrm{Gr}$ & - & - \\
\hline 9. & $\mathrm{D}$ & 8 & 4800 & 8 & 200 & 5 & PCM, Br. Pnia & - & $\mathrm{Gr}$ & D \\
\hline 10. & $\mathrm{M}$ & 6 & 4450 & 7 & - & 6 & PCM & - & - & $\mathrm{D}, \mathrm{Gr}^{*}$ \\
\hline 11. & $\mathrm{H}$ & 4 & 4750 & 8 & 350 & 1 & Convulsion, & $\mathrm{D}, \mathrm{Gr}$ & - & - \\
\hline 12. & $\mathrm{~J}$ & 7 & 6150 & 8 & - & 4 & - & - & $\mathrm{D}$ & $\mathrm{Gr}$ \\
\hline 13. & $\overline{N_{1}}$ & 8 & 4700 & 13 & 650 & 3 & - & $\mathrm{D}, \mathrm{Gr}$ & - & - \\
\hline 14. & $\mathrm{~N}$ & 8 & 6350 & 9 & 300 & 2 & Br. Pnia & $\mathrm{DCr}$ & & \\
\hline 15. & $F$ & $11 / 2$ & 3100 & 4 & - & 4 & - & - & - & $\overline{\mathrm{D}}, \mathrm{Gr}$ \\
\hline 16. & $\mathrm{M}$ & 9 & 6000 & 8 & 700 & 2 & - & $\mathrm{D}, \mathrm{Gr}$ & - & - \\
\hline
\end{tabular}


TABLE 1 (continued).

\begin{tabular}{|c|c|c|c|c|c|c|c|c|c|c|}
\hline \multirow{2}{*}{ No. } & \multirow{2}{*}{ Patient } & \multirow{2}{*}{$\begin{array}{l}\text { Age } \\
(\mathrm{mo})\end{array}$} & \multirow{2}{*}{$\begin{array}{c}\text { Body weight } \\
\text { (B.W:) } \\
\text { on } \\
\text { admission } \\
(\mathrm{gm})\end{array}$} & \multirow{2}{*}{$\begin{array}{l}\text { Duration } \\
\text { of hospita- } \\
\text { lization } \\
\text { (days) }\end{array}$} & \multirow{2}{*}{$\begin{array}{l}\text { Increase } \\
\text { of B.W. } \\
\text { during } \\
\text { hospitali- } \\
\text { zation } \\
\text { (gm) }\end{array}$} & \multirow{2}{*}{$\begin{array}{c}\text { Period } \\
\text { after } \\
\text { which } \\
\text { diarrhoea } \\
\text { stops } \\
\text { (days) } \\
\end{array}$} & \multirow{2}{*}{$\begin{array}{l}\text { Accompanying } \\
\text { disease }\end{array}$} & \multicolumn{3}{|c|}{$R e s u l t s$} \\
\hline & & & & & & & & $\mathrm{E}$ & $\mathrm{G}$ & $\mathbf{P}$ \\
\hline 17. & $\mathrm{H}$ & $51 / 2$ & 5800 & 12 & - & 6 & - & - & - & $\mathrm{D}, \mathrm{Gr}$ \\
\hline 18. & $\mathbf{S}$ & 9 & 8200 & 7 & $5 \overline{00}$ & 3 & - & $\mathrm{D}, \mathrm{Gr}$ & - & - \\
\hline 19. & $\mathrm{H}$ & 7 & 7100 & 7 & - & 1 & - & & - & $\mathrm{Gr}$ \\
\hline 20. & $\mathrm{E}$ & 3 & 4200 & $\dot{\tau}$ & 400 & $\overline{1}$ & Sepsis & - & 一 & - \\
\hline 21. & $\mathbf{L}$ & 1 & 2100 & 12 & 400 & 1 & $\begin{array}{l}\text { Convulsions } \\
\text { Sepsis }\end{array}$ & $\begin{array}{l}\mathrm{D}, \mathrm{Gr} \\
\mathrm{D}, \mathrm{Gr}\end{array}$ & - & - \\
\hline 22. & $N$ & $\begin{array}{l}1 \\
2\end{array}$ & 2700 & 14 & - & $\begin{array}{l}1 \\
9\end{array}$ & Nepat & - & - & $\overline{\mathrm{D}, \mathrm{Gr}}$ \\
\hline 23. & $M$ & 6 & 4750 & 8 & 350 & 1 & $\mathrm{PCM}$ & $\mathrm{D}, \mathrm{Gr}$ & - & - \\
\hline 24. & $\mathrm{E}$ & 24 & 6750 & 7 & 200 & 7 & $\mathrm{PCM}$ & - & $\mathrm{D}, \mathrm{Gr}$ & $\mathrm{Gr}$ \\
\hline 25. & $\overline{\mathrm{S}}$ & 5 & 3550 & 5 & - & 2 & $\begin{array}{l}\text { Down's synd- } \\
\text { rome, VSD }\end{array}$ & - & $\overline{\mathrm{D}}$ & $\overline{\mathrm{Gr}}$ \\
\hline 26. & A & 4 & 4000 & 7 & 300 & 4 & - & $\mathrm{Gr}$ & $\mathrm{D}$ & - \\
\hline 27 . & $\mathrm{S}$ & $21 / 2$ & 3600 & 8 & 350 & 1 & Br. Pnia & $\mathrm{D}, \mathrm{Gr}$ & - & - \\
\hline 28. & $\mathrm{~T}$ & 5 & 4500 & 8 & 400 & 1 & - & $\overrightarrow{\mathrm{D}}, \mathbf{G r}$ & - & 一 \\
\hline 29 . & $\overrightarrow{\mathrm{M}}$ & 1 & 2700 & 8 & 350 & 1 & - & $\mathrm{D}, \mathbf{G r}$ & - & - \\
\hline 30. & $\mathbf{M}$ & $\overline{7}$ & 6200 & 7 & 1050 & 2 & OMA & $\mathrm{D}, \mathrm{Gr}$ & - & - \\
\hline 31. & $\mathrm{H}$ & $61 / 2$ & 5400 & 7 & 350 & 3 & Pharyngitis & $\mathrm{Gr}$ & $\overline{\mathrm{D}}$ & - \\
\hline 32. & $\mathrm{D}$ & $71 / 2$ & 6800 & $\dot{7}$ & 450 & 1 & Pharyngitis & $\mathrm{D}, \mathrm{Gr}$ & - & - \\
\hline 33. & $\mathrm{D}$ & $11^{\prime 2}$ & 7500 & 7 & 500 & $\underline{1}$ & Pharyngitis & $\mathrm{D}, \mathrm{Gr}$ & - & - \\
\hline 34. & A & 18 & 3500 & $i$ & 850 & 1 & PCM, OMA & $\mathrm{D}, \mathbf{G r}$ & - & - \\
\hline 35. & $\mathrm{~S}$ & $21 / 2$ & 2400 & 10 & & 4 & & - & $\mathrm{D}$ & $\mathrm{Gr}$ \\
\hline & $\begin{array}{l}=\mathrm{Dir} \\
=\mathrm{GrC}\end{array}$ & $2 a$ & $\mathrm{ng})$ & $\begin{array}{l}\mathrm{P}=\text { Poor } \\
\mathrm{G} \equiv \text { Good } \\
\mathrm{E} \equiv \text { Exce }\end{array}$ & & & & & & \\
\hline
\end{tabular}


TABLE 1 (continued).

\begin{tabular}{|c|c|c|c|c|c|c|c|c|c|c|}
\hline \multirow{2}{*}{ No. } & \multirow{2}{*}{ Patient } & \multirow{2}{*}{$\begin{array}{l}\text { Age } \\
\text { (mo) }\end{array}$} & \multirow{2}{*}{$\begin{array}{c}\text { Body weight } \\
\text { (B.W.) } \\
\text { on } \\
\text { admission } \\
\text { (gm) }\end{array}$} & \multirow{2}{*}{$\begin{array}{c}\text { Duration } \\
\text { of hospita- } \\
\text { lization } \\
\text { (days) }\end{array}$} & \multirow{2}{*}{$\begin{array}{l}\text { Increase } \\
\text { of B.W. } \\
\text { during } \\
\text { hospitali- } \\
\text { zation } \\
\text { (gm) }\end{array}$} & \multirow{2}{*}{$\begin{array}{l}\text { Period } \\
\text { after } \\
\text { which } \\
\text { diarrhoea } \\
\text { stops } \\
\text { (days) }\end{array}$} & \multirow{2}{*}{$\begin{array}{l}\text { Accompanying } \\
\text { disease }\end{array}$} & \multicolumn{3}{|c|}{$R$ esults } \\
\hline & & & & & & & & $\mathbf{E}$ & G & $P$ \\
\hline 36. & L & 2 & 3100 & 5 & - & 4 & OMA & - & $\mathrm{D}$ & $\mathrm{Gr}$ \\
\hline 37. & $F$ & 8 & 6800 & 7 & 300 & 3 & $\begin{array}{l}\text { OMA, Candi- } \\
\text { diasis }\end{array}$ & $\mathrm{Gr}$ & $\mathrm{D}$ & - \\
\hline 38. & $\mathbf{H}$ & 8 & 8500 & 8 & 1350 & 3 & $\begin{array}{l}\text { Convulsions } \\
\text { OMA }\end{array}$ & $\mathrm{Gr}$ & $\mathrm{D}$ & - \\
\hline 39. & $\mathrm{E}$ & 7 & 6600 & 6 & 50 & 1 & OMA & $D$ & - & $\mathrm{Gr}$ \\
\hline 40. & $\mathbf{Y}$ & 4 & 5500 & 7 & 300 & 3 & Br. Pnia & $\mathrm{Gr}$ & $\mathrm{D}$ & $\ldots$ \\
\hline 41. & $\mathrm{~T}$ & $21 / 2$ & 4360 & 7 & 420 & 3 & OMA & $\mathrm{Gr}$ & $D$ & - \\
\hline
\end{tabular}

$$
\begin{array}{ll}
\mathrm{D}=\text { Diarrhoea (Stopping) } & \mathrm{E} \equiv \text { Excellent } \\
\mathrm{G} x=\mathrm{Growth} & \mathrm{G} \equiv \text { Good } \\
\mathrm{P} \equiv \mathrm{Poor}
\end{array}
$$


not significant. On LLM, 7 (77\%), out of 9 patients showed good to excellent resiults din stopping the diarnhoea but only $4(44 \%)$ showed excellent results in increasing body weight, as judged by the same criteria (Sutedjo et al., 1974).

\section{REFERENCES}

1. DAHLQVIST, A.: Method for assay of intestinal disaccharidases. Analyst. Biochem. $7: 18$ (1964).

2. DAHLQVIST, A., LIMBERG, G, MOEUWISSE, G. and ACKERMAN, M.: Intestinal Dipeptidases and Disaccharidases in children with Malabsorption. Acta Paediatr. Scand. $59: 621$ (1970).

3. MILLER, D. and CRANE, R.K.: The Digestive Function of the Epithelium disaccharide hydrolysis in the isolated border portion of intestinal epithelial cells. Biochem. biophys. Acta 52 : 293 (1961).

4. PLOTKIN, G.R. and ISSELBACHER, K.J.: Secondary Disaccharidase deficiency in adult celiac disease (non tropical sprue) and other malabsorption states. N. Engl. J. Med. 271 : 1033 (1964).

5. SUHARJONO, SUNOTO, ASWITHA DAMAYANTI, SADIKIN DARMAWAN and COTTON, D.G.: Small In- testinal Biopsy in Protein Calorie Mal. nutrition and Coeliac Children. Paediatr. Indones. $11: 7$ (1971) Special Issue.

6. SUNOTO, SUHARTONO and SUTHDJO : Two years study of sugar intolerance in Indonesian Children. Pediatr. Indones. $13: 241$ (1973).

7. SUTEDJO, SUHARJONO, ASWITHA BOEDIARSO, SUNOTO and DADI, E.M.: Effect of Low Lactose Milk (LLIM-Sari Husada) on Refeeding of Diarrhoeal infants. Presented at the First Asian Pediatric Congress, Manila, 30th April - 4th May, 1974.

8. WALKER SMTTH; J.A.: Small bowel morphology in Childhood. Med. J. Aust. 1 : 382 (1969).

9. WALKER SMITH, J.A., PATERSON. H. and KEATING, S. : Evaluation of a New Feeding Formula for Sugar Mallabsorption. Med. J. Aust. 2 : 107 (1973).

10. WALKER SMITH, J.A.: Gastroenteritis. Med. J. Aust. 1 : 329 (1972). 